\title{
NONLINEAR CONTROLLERS FOR TRAJECTORY TRACKING IN BATCH PROCESSES
}

\author{
C. Kravaris, R. A. Wright and J. F. Carrier \\ Department of Chemical Engineering, The University of Michigan, Ann Arbor, MI 48109, U.S.A.
}

(Received 19 May 1988; received for publication 24 June 1988)

\begin{abstract}
Trajectory tracking in nonlinear batch processes is a class of problems where classical transfer function methods fundamentally break down, since no transfer function can represent the system over the entire trajectory. In this work, nonlinear control is proposed as a potential approach for these systems. A general nonlinear control algorithm is derived for systems of relative order one.

The control of batch copolymerization reactors to obtain constant composition is an example which illustrates the use of this method. Simulations for the styrene-acrylonitrile system show the ability of this method to track the required profiles.
\end{abstract}

\section{INTRODUCTION}

Batch processes constitute an important part of chemical engineering control problems. From an engineering viewpoint, the control of batch processes can be divided into two categories: (i) yield optimization problems; and (ii) product quality control problems.

Yield optimization problems are important in the more technologically mature areas of chemical engineering where the production cost is a significant fraction of the selling price (e.g. the petrochemical industry, generic pharmaceuticals, etc.). These are not "pure" control problems, but rather are combined optimization and control problems, or optimal control problems. This class of problems can be formulated as end-point control problems:

$$
\text { given } \begin{aligned}
\dot{x} & =f(x, u), \quad 0 \leqslant t \leqslant t_{f} \\
y & =\Phi\left[x\left(t_{f}\right)\right]
\end{aligned}
$$

find a control law so that either:

(i) $y=$ maximum,

(ii) $y \geqslant a$ desired value.

A special feature of these problems is that the output is not a function of time, but is only a value at a given time instant.

Product quality control problems are especially important in the emerging technologies, such as specialty chemicals, new drugs, etc. where the production cost is a very small fraction of the selling price, and the product has very tight quality specifications, so that it is quite possible to have to throw away an entire batch because the product is off-spec. Throwing away a batch usually means a lot of money because the production is small-scale and the selling price of the product is very high. Product quality problems are also important in the technologically mature processes, although frequently they are not crucial due to looser product specifications. These can frequently be formulated as tracking problems:

$$
\text { given } \quad \begin{aligned}
& \dot{x}=f(x, u), \\
& y=h(x),
\end{aligned}
$$

find a control law so that $y(t)$ tracks a given trajectory $y_{\mathrm{sp}}(t)$.

It is possible to reformulate an end-point problem of the form (1) as a tracking problem by applying Pontryagin's principle. The problem then reduces to one of designing a control law that will force the system to track the optimal profiles $x(t)$. This approach worked well in a number of cases including penicillin production (Constandinides et al., 1970).

However, modeling error, including disturbances, introduces error in the calculation of optimal profiles. If the modeling error is not significant, the profiles will be suboptimal, but will still provide a significant increase in yield. In the presence of significant modeling error, end-point problems must be formulated as adaptive optimization problems.

For batch process control integral transform methods, in general, break down, since there is no transfer function to describe the system over the entire trajectory due to nonlinearities. Most of the literature on batch process control concerns batch reactors, for which there are typically three phases: (i) a start-up phase where reactor contents are brought from room temperature to the desirable reaction tempeature; (ii) a reaction phase where a temperature setpoint, which may be constant or time-varying, is tracked; and (iii) a shut-down phase where the mixture is cooled down to room temperature. A survey of the major advances in batch process control is given in Juba and Hamer (1986). Marroquin and Luyben (1973) derived seventh- to tenth-order 
models for their batch reactor and (Marroquin and Luyben, 1972) also evaluated a nonlinear cascade controller, where one of the controller gains was dependent on the error in one of the loops, to maintain isothermal operation. Clarke and Gawthrop (1981) and Kiparissides and Shah (1983) used adaptive control for startup, isothermal operation, and then shutdown. Jutan and Uppal (1984) use a feedforward-feedback nonlinear controller where the feedforward part estimated the heat released by reaction for startup and isothermal operation. A similar idea was explored by $\mathbf{W u}$ (1985). Tirrel and Gromley (1981) calculate a temperature profile for constant composition in a batch copolymerization reactor, but do not study the problem of tracking that profile by manipulating the heat input.

The objective of this work is to propose a general nonlinear feedforward-feedback control methodology for set-point tracking in batch processes of relative order one. The composition control of a copolymerization batch reactor will serve as a paradigm to meet this goal. This was chosen because it is an industrially important genuine tracking problem for product quality control. When the control problem is formulated as tracking the TirrelGromley temperature profile (Tirrel and Gromley, 1981), the feedforward part provides the estimated heat of reaction. When the control problem is formulated in terms of composition, the feedforward part provides an estimate of the temperature gradient required for constant composition and the estimated heat of reaction.

The feedforward-feedback methodology proposed in this paper is equivalent to the globally linearizing control (GLC) structure with a reduced-order openloop observer, introduced by Kravaris and Chung (1987). The GLC structure has arisen from differential geometric nonlinear systems theory; the present work can be viewed as a feedforward-feedback interpretation of GLC.

\section{A NONLINEAR FEEDFORWARD-FEEDBACK CONTROL APPROACH}

(a) The output of the system is one of the states Consider the nonlinear dynamic system:
Process:

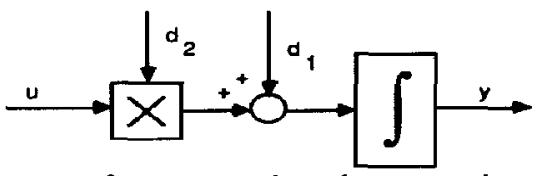

Fig. 1. Block diagram of a system when the output is one of the states.

$$
\begin{aligned}
x_{1} & =f_{1}\left(x_{1}, \ldots, x_{n-1}, x_{n}\right)+g_{1}\left(x_{1}, \ldots, x_{n-1}, x_{n}\right) u \\
\vdots & \\
\dot{x}_{n-1} & =f_{n-1}\left(x_{1}, \ldots, x_{n-1}, x_{n}\right)+g_{n-1}\left(x_{1}, \ldots, x_{n-1}, x_{n}\right) u \\
\dot{x}_{n} & =f_{n}\left(x_{1}, \ldots, x_{n-1}, x_{n}\right)+g_{n}\left(x_{1}, \ldots, x_{n-1}, x_{n}\right) u \\
y & =x_{n}
\end{aligned}
$$

where

$$
g_{n}\left(x_{1}, \ldots, x_{n-1}, x_{n}\right) \neq 0 .
$$

This system can also be viewed as a first-order system with $x_{1}, \ldots, x_{n-1}$ as disturbances:

$$
\dot{y}=f_{n}\left(x_{1}, \ldots, x_{n-1}, y\right)+g_{n}\left(x_{1}, \ldots, x_{n-1}, y\right) u \text {. }
$$

This may also be written as (see Fig. 1):

$$
\dot{y}=d_{1, \text { overall }}+d_{2, \text { overall }} u,
$$

where:

$$
\begin{aligned}
& d_{1, \text { overatl }}=f_{n}\left(x_{1}, \ldots, x_{n-1}, y\right), \\
& d_{2, \text { overall }}=g_{n}\left(x_{1}, \ldots, x_{n-1}, y\right) .
\end{aligned}
$$

Thus, although the system may consist of many state equations, the "effective" order is the relative order which is equal to one.

If the system is initially at the set-point trajectory $y=y_{\mathrm{sp}}(t)$ at $t=t_{0}$ and $d_{1, \text { overall }}$ and $d_{2 \text {, overall can be }}$ measured or computed exactly, then the feedforward control law:

$$
u=\frac{\mathrm{d} y_{\mathrm{sp}} / \mathrm{d} t-d_{1, \text { overall }}}{d_{2, \text { overall }}},
$$

will keep it along the set point trajectory for all $t \geqslant t_{0}$ (see Fig. 2).

Of course, $d_{1 \text {, overal }}$ and $d_{2, \text { overall will not be measured }}$ on-line, but will have to be computed from on-line measurements or estimates of $x_{1}, \ldots, x_{n-1}$. This leads to the following control law:

$$
u=\frac{\mathbf{d} y_{\mathrm{sp}} / \mathrm{d} t-f_{n}\left(\hat{x}_{1}, \ldots, \hat{x}_{n-1}, y\right)}{g_{n}\left(\hat{x}_{1}, \ldots, \hat{x}_{n-1}, y\right)},
$$

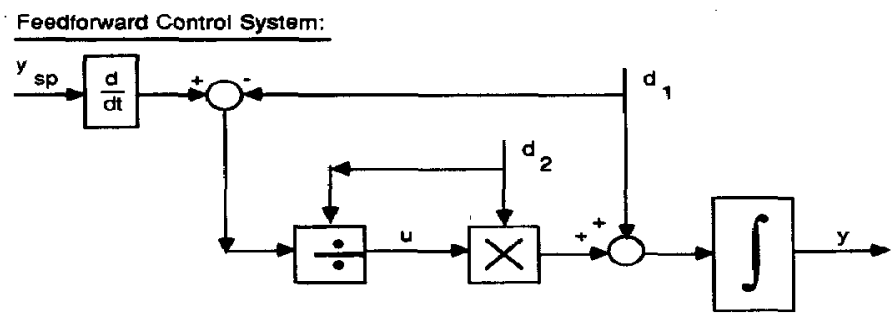

Fig. 2. Feedforward loop for control of system in Fig. 1. 
where $\hat{x}_{1}, \ldots, \hat{x}_{n-1}$ represent either measurements or on line estimates of $x_{1}, \ldots, x_{n-1}$ from:

$$
\begin{aligned}
& \dot{x}_{1}= f_{1}\left(\hat{x}_{1}, \ldots, \hat{x}_{n-1}, y\right)+g_{1}\left(\hat{x}_{1}, \ldots, \hat{x}_{n-1}, y\right) u \\
& \vdots \\
& \dot{x}_{n-1}= f_{n-1}\left(\hat{x}_{1}, \ldots, \hat{x}_{n-1}, y\right) \\
&+g_{n-1}\left(\hat{x}_{1}, \ldots, \hat{x}_{n-1}, y\right) u .
\end{aligned}
$$

The control law (7) together with (8) can have the following interpretation. The set of equations:

$$
\begin{aligned}
\dot{x}_{1}= & f_{1}\left(\hat{x}_{1}, \ldots, \hat{x}_{n-1}, y\right)+g_{1}\left(\hat{x}_{1}, \ldots, \hat{x}_{n-1}, y\right) u \\
& \vdots \\
\dot{x}_{n-1}= & f_{n-1}\left(\hat{x}_{1}, \ldots, \hat{x}_{n-1, y)}\right. \\
& +g_{n-1}\left(\hat{x}_{1}, \ldots, \hat{x}_{n-1}, y\right) u, \\
d_{1, \text { overall }}= & f_{n}\left(\hat{x}_{1}, \ldots, \hat{x}_{n-1}, y\right), \\
d_{2, \text { overall }}= & g_{n}\left(\hat{x}_{1}, \ldots, x_{n-1}, y\right),
\end{aligned}
$$

can be viewed as a disturbance model to the process (5). According to the internal model principle, the control law must provide an internal model of each disturbance at the point that it enters the control loop. This is exactly what the control law is doing. Of course, the estimation of $d_{1 \text {, overall }}$ and $d_{2 \text {, overall will }}$ always have error due to model uncertainty. For this reason, the above feedforward control-disturbance estimator methodology will not work unless it is combined with a feedback controller.

For example, the feedback controller can he PID, in which case (see Fig. 3) (b) Special case-computed heat of reaction method for temperature control of a chemical reactor

For the special case of temperature control in a chemical reactor, the system may be represented as:

$$
\begin{aligned}
\dot{x}_{1} & =f_{1}\left(x_{1}, \ldots, x_{n-1}, x_{n}\right) \\
& \vdots \\
\dot{x}_{n-1} & =f_{n-1}\left(x_{1}, \ldots, x_{n-1}, x_{n}\right), \\
\dot{x}_{n} & =\gamma\left\{f_{n}\left(x_{1}, \ldots, x_{n-1}, x_{n}\right)+u\right\}, \\
y & =x_{n},
\end{aligned}
$$

where

- $x_{1}, \ldots, x_{n-1}$ are concentrations

- $x_{n}$ is the temperature

- The first $n-1$ equations are component mass balances

- The $n$th equation is the energy balance

- $u$ is the heat added or removed by the heat exchanger

- $\gamma$ is a constant

- $f_{n}\left(x_{1}, \ldots, x_{n-1}, x_{n}\right)$ is the heat released or absorbed due to the reaction.

The control law (11) will become:

$$
u=\mathbf{P I D}+\frac{1}{\gamma} \frac{\mathrm{d} y_{\mathrm{sp}}}{\mathrm{d} t}-f_{n}\left(\hat{x}_{1}, \ldots, \hat{x}_{n-1}, y\right) .
$$

It is clear that this control law feeds forward the heat of reaction. This is somewhat reminiscent of the Extensive Variable Control of Georgakis (1986) in

$$
u=\frac{K_{\mathrm{c}}\left[\left(y_{\mathrm{sp}}-y\right)+1 / \tau_{1} \int\left(y_{\mathrm{sp}}-y\right)+\mathbf{d} / \mathrm{d} t\left(y_{\mathrm{sp}}-y\right)\right]+\mathrm{d} y_{\mathrm{sp}} / \mathbf{d} t-d_{1, \text { overall }}}{d_{2, \text { overall }}}
$$

This leads to:

$$
u=\frac{K_{c}\left[\left(y_{\mathrm{sp}}-y\right)+1 / \tau_{1} \int\left(y_{\mathrm{sp}}-y\right)+\mathrm{d} / \mathrm{d} t\left(y_{\mathrm{sp}}-y\right)\right]+\mathrm{d} y_{\mathrm{sp}} / \mathrm{d} t-f_{n}\left(\hat{x}_{1}, \ldots, \hat{x}_{n-1}, y\right)}{g_{n}\left(\hat{x}_{1}, \ldots, \hat{x}_{n-1}, y\right)} .
$$

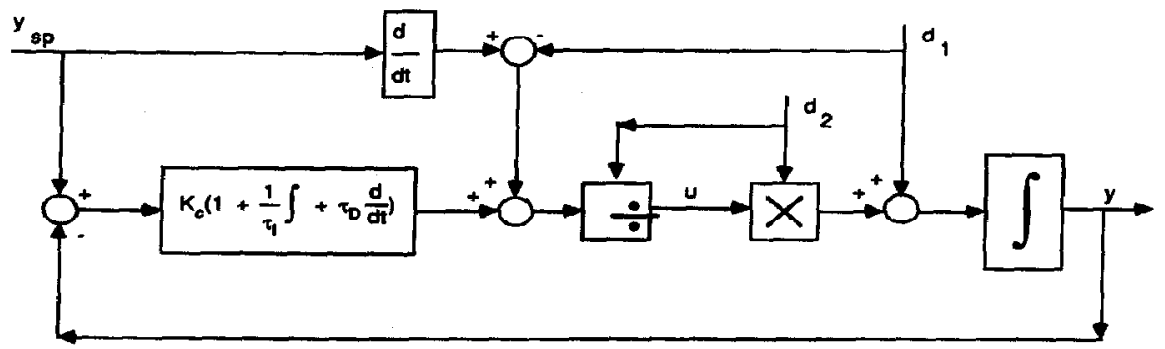

Fig. 3. Feedforward-feedback control of system in Fig. 1. 
that an extensive variable is used by the controller. However, in Extensive Variable Control, the variable fed back is chosen to be an extensive variable. Here, the quantity fed forward, as described in the previous derivation, turns out to be an extensive variable.

(c) The output of the system is an arbitrary function of the states

Consider the nonlinear dynamic system:

$$
\begin{aligned}
\dot{x}_{1} & =f_{1}\left(x_{1}, \ldots, x_{n-1}, x_{n}\right)+g_{1}\left(x_{1}, \ldots, x_{n-1}, x_{n}\right) u, \\
\vdots & \\
\dot{x}_{n-1} & =f_{n-1}\left(x_{1}, \ldots, x_{n-1}, x_{n}\right)+g_{n-1}\left(x_{1}, \ldots, x_{n-1}, x_{n}\right) u, \\
\dot{x}_{n} & =f_{n}\left(x_{1}, \ldots, x_{n-1}, x_{n}\right)+g_{n}\left(x_{1}, \ldots, x_{n-1}, x_{n}\right) u \\
y & =h\left(x_{1}, \ldots, x_{n-1}, x_{n}\right) .
\end{aligned}
$$

Let $x_{n}$ be a state variable that $y$ depends on, i.e:

$$
\frac{\partial h}{\partial x_{n}}\left(x_{1}, \ldots, x_{n-1}, x_{n}\right) \neq 0
$$

and denote by $x_{n}=H\left(x_{1}, \ldots, x_{n-1}, y\right)$ the implicit function defined as the solution of $y=$ $h\left(x_{1}, \ldots, x_{n-1}, x_{n}\right)$. Then the above system is equivalent to:

$$
\begin{aligned}
\dot{x}_{1}= & f_{1}\left[x_{1}, \ldots, x_{n-1}, H\left(x_{1}, \ldots, x_{n-1}, y\right)\right] \\
& +g_{1}\left[x_{1}, \ldots, x_{n-1}, H\left(x_{1}, \ldots, x_{n-1}, y\right)\right] u \\
\dot{x}_{n-1}= & f_{n-1}\left[x_{1}, \ldots, x_{n-1}, H\left(x_{1}, \ldots, x_{n-1}, y\right)\right] \\
& +g_{n-1}\left[x_{1}, \ldots, x_{n-1}, H\left(x_{1}, \ldots, x_{n-1}, y\right)\right] u \\
\dot{y}= & \left(\sum_{i=1}^{n} \frac{\partial h}{\partial x_{i}} f_{i}\right)+\left(\sum_{i=1}^{n} \frac{\partial h}{\partial x_{i}} g_{i}\right) u .
\end{aligned}
$$

Under the assumption that:

$$
\sum_{i=1}^{n} \frac{\partial h}{\partial x_{i}} g_{l} \neq 0
$$

the necessary control law is given by:

\section{(d) Limitations-systems of relative order one}

A dynamic system of the form (14) for which:

$$
\sum_{i=1}^{n} \frac{\partial h}{\partial x_{i}} g_{i} \neq 0
$$

is said to be of relative order one. For such a system:

$$
\dot{y}=\left(\sum_{i=1}^{n} \frac{\partial h}{\partial x_{i}} f_{i}\right)+\left(\sum_{i=1}^{n} \frac{\partial h}{\partial x_{i}} g_{i}\right) u
$$

Hence $\mathrm{d} y / \mathrm{d} t$ explicitly depends on $u$. For the special case where $y=x_{n}$, i.e. one of the states, then a relative order of one means that $g_{n} \neq 0$. Clearly the nonlinear feedforward-feedback control method presented above is restricted to systems of relative order one.

However, systems of relative order one arise naturally in chemical process control. A relative order of : one means that the manipulated input directly affects the first derivative of the output. A simple example of this is when the temperature of a batch reactor is being controlled by manipulating the heat input to the system. Since the first derivative of the output is the energy balance, it is clear that this must be affected by the manipulated input. $A$ system of relative order $r$ is the one for which $\mathrm{d} y / \mathrm{d} t, \ldots, d^{r-1} y / \mathrm{d} t^{r-1}$ are not explicitly dependent on $u$, whereas $\mathrm{d}^{r} y / \mathrm{d} t^{r}$ is an explicit function of $u$. A relative order of $n$ means that the manipulated input affects only the $n$th derivative of the output. In that case the states which are explicitly represented in the output are not affected by the manipulated input, and the states which directly affect those states are not affected by the manipulated input, etc. for $n$ digressions. Thus the relative order may be thought of as the number of state equations which must be considered, starting from those states appearing directly in the output, until one is found with the manipulated input on the right-hand-side. Systems of relative order one will be less sluggish than those of higher relative order. Therefore, in systems for which the choice of manipulated input is, by heuristic considerations, "good" will usually be of low relative

$$
u=\frac{\frac{\mathrm{d} y_{\mathrm{sp}}}{\mathrm{d} t}-\sum_{i=1}^{n} \frac{\partial h}{\partial x_{i}}\left[\hat{x}_{1}, \ldots, \hat{x}_{n-1}, H\left(\hat{x}_{1}, \ldots, \hat{x}_{n-1}, y\right)\right] f_{i}\left[\hat{x}_{1}, \ldots, \hat{x}_{n-1}, H\left(\hat{x}_{1}, \ldots, \hat{x}_{n-1}, y\right)\right]+\text { PID }}{\sum_{i=1}^{n} \frac{\partial h}{\partial x_{i}}\left[\hat{x}_{1}, \ldots, \hat{x}_{n-1}, H\left(\hat{x}_{1}, \ldots, \hat{x}_{n-1}, y\right)\right] g_{i}\left[\hat{x}_{1}, \ldots, \hat{x}_{n-1}, H\left(\hat{x}_{1}, \ldots, \hat{x}_{n-1}, y\right)\right],}
$$

where $\hat{x}_{1}, \ldots, \hat{x}_{n-1}$ are computed by solving:

$$
\begin{aligned}
\dot{x}_{1}= & f_{1}\left[\hat{x}_{1}, \ldots, \hat{x}_{n-1}, H\left(\hat{x}_{1}, \ldots, \hat{x}_{n-1}, y\right)\right] \\
& +g_{1}\left[\hat{x}_{1}, \ldots, \hat{x}_{n-1}, H\left(\hat{x}_{1}, \ldots, \hat{x}_{n-1}, y\right)\right] u, \\
& \vdots \\
\dot{x}_{n-1}= & f_{n-1}\left[\hat{x}_{1}, \ldots, \hat{x}_{n-1}, H\left(\hat{x}_{1}, \ldots, \hat{x}_{n-1}, y\right)\right] \\
& +g_{n-1}\left[\hat{x}_{1}, \ldots, \hat{x}_{n-1}, H\left(\hat{x}_{1}, \ldots, \hat{x}_{n-1}, y\right)\right] u .
\end{aligned}
$$

order. For example, if an $\boldsymbol{m}$-stage distillation tower were the system, and it was required to control the composition on the top tray, one would not normally choose the heat added to the reboiler as the manipulated input if something further up the tower were available, since this would lead to an extremely sluggish response and poor control system performance. This is because the relative order of the system, if such a choice were made would be $r=2 m=n$. 


\section{NONLINEAR COMPOSITION CONTROL OF BATCH COPOLYMERIZATION}

The system chosen to illustrate this control approach was the batch copolymerization of styrene and acrylonitrile using xylene as a solvent (Tirrel and Gromley, 1981). One particular problem that is frequently encountered in batch copolymerization systems is composition drift. This occurs when all monomers do not react with the polymer at the same rate and constant temperature is maintained; the composition of the copolymer drifts away from its initial value as the monomer mixture becomes depleted in the more reactive monomer. This can significantly effect the properties of the copolymer. To solve this problem, Tirrel and Gromley (1981) have computed temperature profiles to maintain constant polymer composition in a batch reactor. Another alternative is to use a semibatch reactor and vary the flowrate of the more reactive monomer while keeping the temperature constant. Control laws for both alternatives will be explored.

\section{(a) Batch case}

Under the assumptions of free-radical copolymerization, applicability of the quasi-steady state hypothesis and absence of the gel effect, the governing equations for copolymerization in a batch reactor are:

$$
\begin{aligned}
R_{i} & =k_{\mathrm{D} 1}[I] \\
\phi & =k_{t 12} /\left[2\left(k_{t 11} k_{t 22}\right)^{1 / 2}\right] \\
R_{\mathrm{p}} & =\frac{\left(r_{1}\left[\mathrm{M}_{1}\right]^{2}+2\left[\mathrm{M}_{1}\right]\left[\mathrm{M}_{2}\right]+r_{2}\left[\mathrm{M}_{2}\right]^{2}\right)\left(R_{i}\right)^{1 / 2}}{\left(\xi_{1}^{2}\left[\mathrm{M}_{1}\right]^{2}+2 \phi \xi_{1} \xi_{2}\left[\mathrm{M}_{1}\right]\left[\mathrm{M}_{2}\right]+\xi_{2}^{2}\left[\mathrm{M}_{2}\right]^{2}\right)^{1 / 2}}
\end{aligned}
$$

$F_{1}$ is the mol fraction of monomer 1 added to the copolymer chain

To simplify the derivation of the control law, the system may be written as:

$$
\begin{aligned}
\frac{\mathrm{d}\left[\mathbf{M}_{1}\right]}{\mathrm{d} t} & =\mathrm{F}_{1}\left(\left[\mathrm{M}_{1}\right], \zeta_{1}, T\right), \\
\frac{\mathrm{d} \zeta_{1}}{\mathrm{~d} t} & =F_{2}\left(\zeta_{1}, T\right), \\
\frac{\mathrm{d} T}{\mathrm{~d} t} & =F_{3}\left(\left[\mathbf{M}_{1}\right], \zeta_{1}, T\right)+u, \\
\mathrm{~F}_{1} & =h\left(\zeta_{1}, T\right) .
\end{aligned}
$$

The control objective is to keep $F_{1}$ constant throughout, given on-line measurements of the temperature only, as it is very difficult in practice to accurately measure $F_{1}$ on-line. This may be accomplished in one of two ways. First, $F_{1}$ may be estimated on-line and a control law may be formulated using the error between $F_{1 s p}$ and $\hat{F}_{1}$, where $\hat{F}_{1}$ is the on-line estimate, to manipulate the heat added to the system, $Q$. The second method is to first solve off-line for the

$$
\begin{aligned}
\frac{\mathrm{d}\left[\mathbf{M}_{1}\right]}{\mathrm{d} t} & =\frac{-\left(r_{1}\left[\mathbf{M}_{1}\right]^{2}+\left[\mathbf{M}_{1}\right]\left[\mathbf{M}_{2}\right]\right)\left(R_{i}\right)^{1 / 2}}{\left(\xi_{1}^{2}\left[\mathbf{M}_{1}\right]^{2}+2 \phi \xi_{1} \xi_{2}\left[\mathbf{M}_{1}\right]\left[\mathbf{M}_{2}\right]+\xi_{2}^{2}\left[\mathbf{M}_{2}\right]^{2}\right)^{1 / 2}}, \\
\frac{\mathrm{d} \zeta_{1}}{\mathrm{~d} t} & =\frac{\left[\left(r_{1}+r_{2}-2\right) \zeta_{1}^{3}+\left(3-r_{1}-2 r_{2}\right) \zeta_{1}^{2}+\left(r_{2}-1\right) \zeta_{1}\right]\left(R_{t}\right)^{1 / 2}}{\left(\xi_{1} \xi_{2}\left[\left(\psi_{1}-2 \phi+\psi_{2}\right) \zeta_{1}^{2}+2\left(\phi-\psi_{2}\right) \zeta_{1}+\psi_{2}\right]\right)^{1 / 2}},
\end{aligned}
$$

$$
\begin{aligned}
\frac{\mathrm{d} T}{\mathrm{~d} t} & =\frac{-\Delta H_{\mathrm{cop}} R_{\mathrm{p}}}{C_{\mathrm{pm}}}+\frac{Q}{C_{\mathrm{pm}} V}, \\
\mathrm{~F}_{1} & =\frac{r_{1} \zeta_{1}^{2}+\zeta_{1} \zeta_{2}}{r_{1} \zeta_{1}^{2}+2 \zeta_{1} \zeta_{2}+r_{2} \zeta_{2}^{2}},
\end{aligned}
$$

temperature profile which will keep $F_{1}=F_{1 s p}$ using the method of Tirrel and Gromley (1981). Then a control law must be found to track this profile by manipulating $Q$. The temperature profile, $T=T_{\mathrm{sp}}(t)$, is found by first solving (22) with respect to $\zeta_{1}$ for $F_{1}=F_{1 s p}$ :

$$
\zeta_{1}=\frac{1-2 F_{1 \mathrm{sp}}\left(1-r_{2}\right)-\left[4 F_{1 \mathrm{sp}}\left(r_{1} r_{2}-1\right)\left(1-F_{1 s \mathrm{p}}\right)+1\right]^{1 / 2}}{2\left(\mathrm{~F}_{1 \mathrm{sp}}\left(r_{1}+r_{2}-2\right)-r_{1}+1\right)}=W(T)
$$

where:

$$
\begin{aligned}
& r_{1}=k_{p 11} / k_{p 12}=r_{10} \exp \left[-\Delta E_{1} / R\left(1 / T-1 / T_{\text {ref }}\right)\right] \\
& r_{2}=k_{p 22} / k_{p 21}=r_{20} \exp \left[-\Delta E_{2} / R\left(1 / T-1 / T_{\text {ref }}\right)\right] \\
& \xi_{1}=\left(2 k_{i 11} / k_{p 11}^{2}\right)^{1 / 2} r_{1} \\
& \xi_{2}=\left(2 k_{t 22} / k_{p 22}^{2}\right)^{1 / 2} r_{2} \\
& \zeta_{2}=1-\zeta_{1} \\
& \psi_{1}=\xi_{1} / \xi_{2} \\
& \psi_{2}=\xi_{2} / \xi_{1}
\end{aligned}
$$

and then integrating the ordinary differential equation:

$$
\frac{\mathrm{d} T}{\mathrm{~d} t}=\frac{F_{2}[W(T), T]}{\mathrm{d} W(T) / \mathrm{d} T}
$$

It is interesting to note that an equivalent form to (38) is:

$$
\frac{\mathrm{d} T}{\mathrm{~d} t}=\frac{-\mathbf{F}_{2} \partial h / \partial \zeta_{1}}{\partial h / \partial T}
$$

which is a result of the implicit function theorem. 
From the discussion in the previous section and (17) it is straightforward to show that the control law obtained when the problem is formulated with $F_{1}$ as the output is:

$$
u=-\frac{\partial h / \partial \zeta_{1} \mathbf{F}_{2}}{\partial h / \partial T}-\mathbf{F}_{3}+\frac{\operatorname{PID}\left(\mathbf{F}_{\text {lap }}-\hat{F}_{1}\right)}{\partial h / \partial T}
$$

where the states $\left[M_{1}\right](t)$ and $\zeta_{1}(t)$ are estimated on-line from (19) and (20) and the output $F_{1}(t)$ is estimated from (22), given on-line temperature measurements. Similarly, if it is desired to track the computed temperature profile, the control law is [from (11) or (13)]:

$$
u=\frac{\mathrm{d} T_{\mathrm{sp}}}{\mathrm{d} t}-\mathbf{F}_{3}+\operatorname{PID}\left(T_{\mathrm{sp}}-T\right),
$$

where the states $\left[M_{1}\right](t)$ and $\zeta_{1}(t)$ are again estimated on-line from (19) and (20) given the on-line temperature measurements. It is clear that although the control laws obtained by both approaches are similar, they are not equivalent, since the first term of $(40)$ is equal to the first term of (41) only when the current temperature is on the desired profile, i.e. $T=T_{\mathrm{sp}}(t)$. The control laws of the both alternatives can be interpreted as doing the following:

- The first term provides an estimate of the temperature gradient necessary for constant composition.

- The second term cancels the heat of reaction.

- The third term provides PID feedback.

\section{(b) Semibatch case}

Consider the case where monomer 1 is continuously fed to the reactor. The inlet flowrate will be manipulated to control the copolymer composition under isothermal conditions. The governing equations under the same assumptions as in the batch case are:

$$
\begin{aligned}
& \frac{\mathrm{d}\left[\mathbf{M}_{1}\right]}{\mathrm{d} t}=\frac{-\left(r_{1}\left[\mathbf{M}_{1}\right]^{2}+\left[\mathbf{M}_{1}\right]\left[\mathbf{M}_{2}\right]\right)\left(R_{i}\right)^{1 / 2}}{\left(\xi_{1}^{2}\left[\mathbf{M}_{1}\right]^{2}+2 \phi \xi_{1} \xi_{2}\left[\mathbf{M}_{1}\right]+\xi_{2}^{2}\left[\mathbf{M}_{2}\right]^{2}\right)^{1 / 2}} \\
& -\frac{F_{i}}{V}\left(\left[M_{1}\right]-C_{M_{1}}\right) \\
& \mathrm{F}_{1}=\frac{r_{1} \zeta_{1}^{2}+\zeta_{1} \zeta_{2}}{r_{1} \zeta_{1}^{2}+2 \zeta_{1} \zeta_{2}+r_{2} \zeta_{2}^{2}}
\end{aligned}
$$

$$
\frac{\mathrm{d} \zeta_{1}}{\mathrm{~d} t}=\frac{\left[\left(r_{1}+r_{2}-2\right) \zeta_{1}^{3}+\left(3-r_{1}-2 r_{2}\right) \zeta_{1}^{2}+\left(r_{2}-1\right) \zeta_{1}\right]\left(R_{i}\right)^{1 / 2}}{\left\{\xi_{1} \xi_{2}\left[\left(\psi_{1}-2 \phi+\psi_{2}\right) \zeta_{1}^{2}+2\left(\phi-\psi_{2}\right) \zeta_{1}+\psi_{2}\right]\right\}^{1 / 2}}+\zeta_{1} \zeta_{2} \frac{C_{\mathbf{M}_{1}} F_{i}}{\left[\mathbf{M}_{1}\right] V}
$$

To simplify the derivation of the control law, the system may be written as:

$$
\begin{aligned}
\frac{\mathrm{d}\left[\mathbf{M}_{1}\right]}{\mathrm{d} t} & =\mathbb{F}_{1}\left(\left[\mathrm{M}_{1}\right], \zeta_{1}\right)+\mathbb{G}_{1}\left(\left[\mathrm{M}_{1}\right]\right) u, \\
\frac{\mathrm{d} \zeta_{1}}{\mathrm{~d} t} & =\mathbb{F}_{2}\left(\zeta_{1}\right)+\mathbb{G}_{2}\left(\left[\mathbf{M}_{1}\right], \zeta_{1}\right) u, \\
F_{1} & =\operatorname{h}\left(\zeta_{1}\right) .
\end{aligned}
$$

The control law for such a system is:

$$
u=-\frac{\mathbb{F}_{2}}{\mathbb{G}_{2}}+\frac{\operatorname{PID}\left(F_{1 s p}-\hat{F}_{1}\right)}{G_{2} \partial \mathrm{lt} / \partial \zeta_{1}}
$$

Both cases have important practical limitations. In the batch case it is not always possible to find a temperature profile that guarantees uniform composition of the copolymer [see Ray and Gall (1969) for necessary conditions]. In the semibatch case adding one monomer continuously can lead to difficult mixing operations if the polymerizing mass is very viscous. One possibility for future consideration may be to manipulate both temperature and inlet flowrate for a semibatch reactor in a coordinated way, as suggested in Tsoukas et al. (1982).

\section{SIMULATION RESULTS}

The process parameters used for the simulations are summarized in Table 1 . Simulations were performed for only the batch case where both errors in the initial values of $\left[M_{1}\right]$ and $\zeta_{1}$ and noise in the heat added were considered as disturbances. Both control laws, (40) and (41), were simulated. In all cases the control objective was to keep $F_{1}=0.62$. The temperature profile which results in a constant composition is shown in Fig. 4. In all simulation runs the initial temperature was taken to be $25^{\circ} \mathrm{C}$, to simulate startup. In the simulations, conventional PI was chosen as a feedback controller for its simplicity. It was beyond the scope of this work to calculate "best possible" PI controller settings. The results below show the response for controller gains found by trial and error.
In all the simulations the initial values of the state estimator were set equal to the nominal initial values. This is because, in practice, a run would not be started unless the operator thinks that the reactor is charged with the right amounts of monomers. Of course, the actual initial values of the states $\left[M_{1}\right]$ and $\zeta_{1}$ can be in error and these are disturbances to the system.

Figures 5-12 show the resulting temperature profile and $F_{1}$ and $\hat{F}_{1}$ profiles for four different cases using 
Table 1

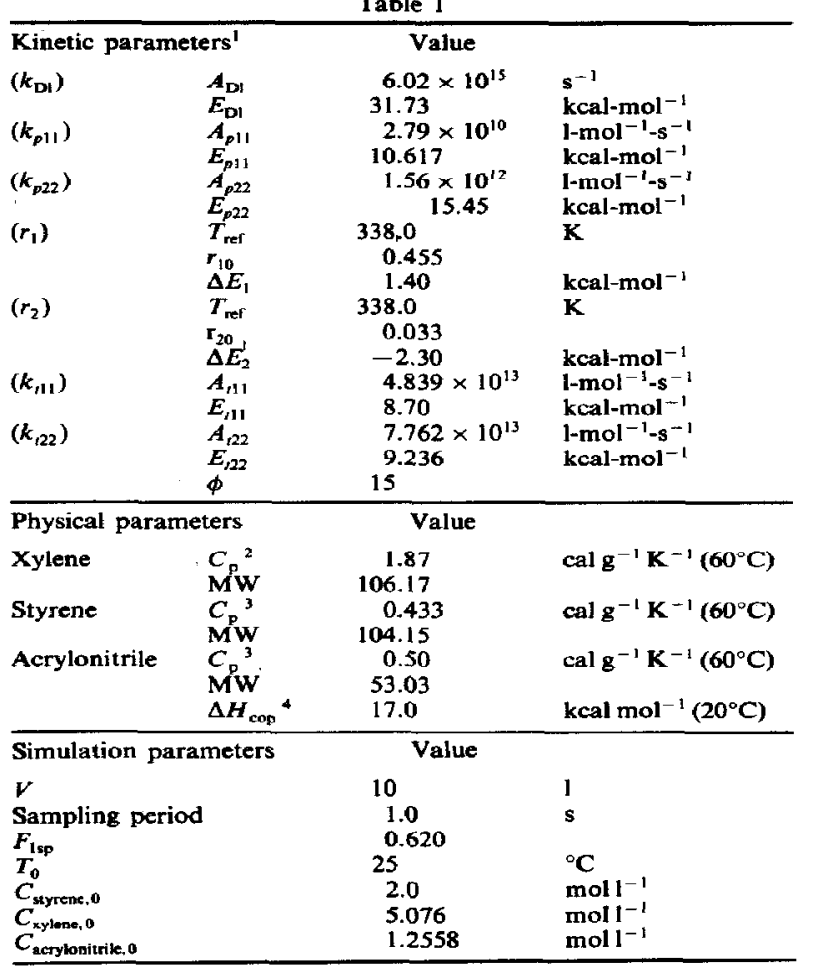

'From Tirrel and Gromley (1981).

${ }^{2}$ From Touloukian and Ho (1976)

${ }^{3}$ From Blout (1949).

${ }^{4}$ From Miyama (1961).

the control law in terms of $F_{1}$ [equation (40)]. For all four cases $K_{c}=50$ and $\tau_{I}=500$. In the first case (Figs 5 and 6) there were no disturbances. Obviously the profiles for $F_{1}$ and $\hat{F}_{1}$ are identical in this simulation. In the second trial (Figs 7 and 8 ) a $1 \%$ error was introduced in the initial condition of $\zeta_{1}$. Note that there was an offset in the response. This is because an error in $\zeta_{1}(0)$ introduces an error into the copolymer composition, $F_{1}$, which cannot be detected, since $T$ is the only measured output. However, over the course of a run the composition drifted only slightly when

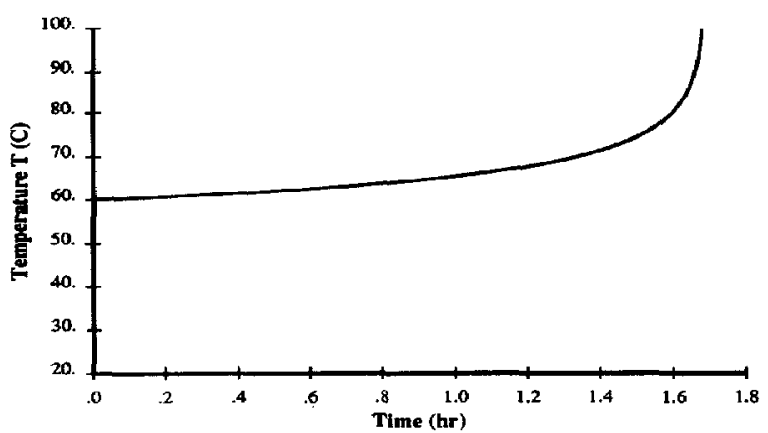

Fig. 4. Temperature profile which will result in constant composition.

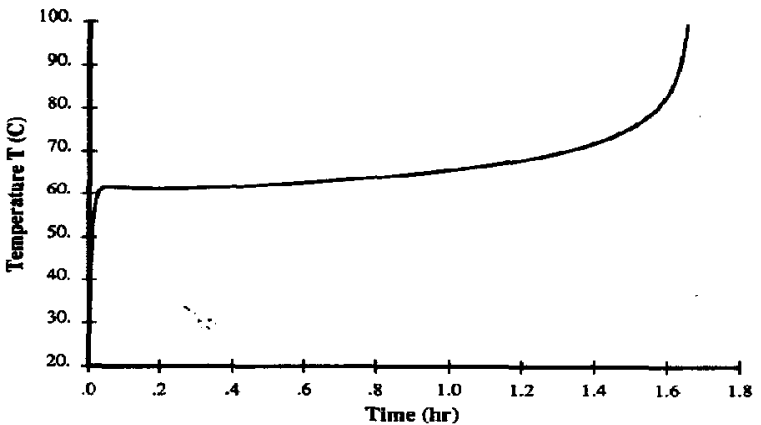

Fig. 5. Temperature profile using control law in terms of $F_{\mathbf{l}}$ [equation (40)] with no disturbance.

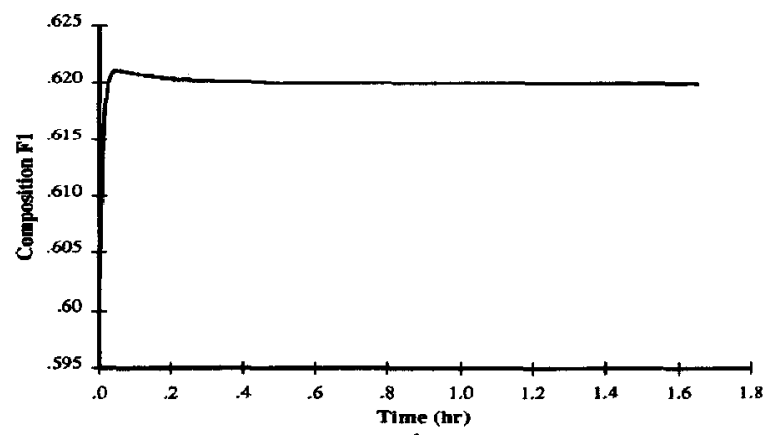

Fig. 6. $F_{1}$ and $F_{1}$ profiles using control law in terms of $F_{1}$ [equation (40)] with no disturbance.

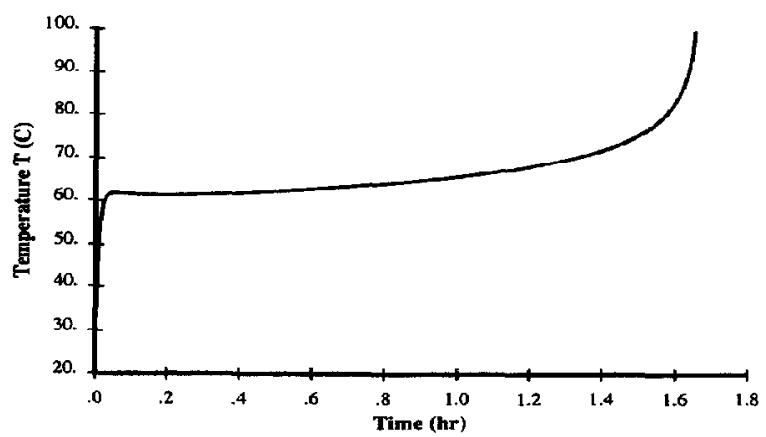

Fig. 7. Temperature profile using control law (40) with $1 \%$ error in $\zeta_{1}(0)$.

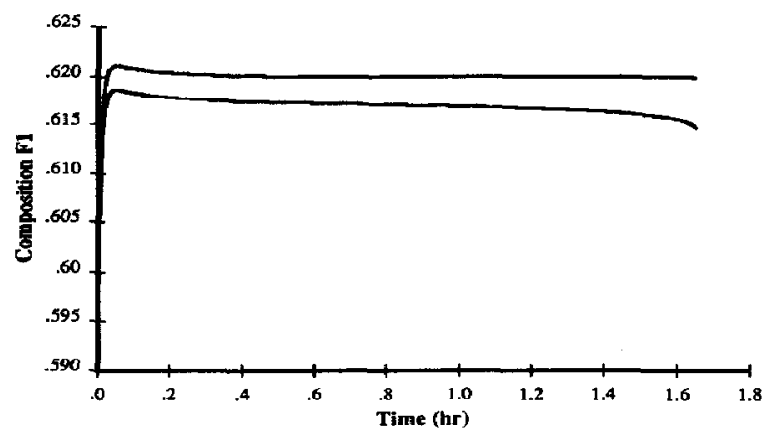

Fig. 8. $F_{1}$ and $\hat{F}_{1}$ profiles using control law (40) with $1 \%$ error in $\zeta_{1}(0)$. 


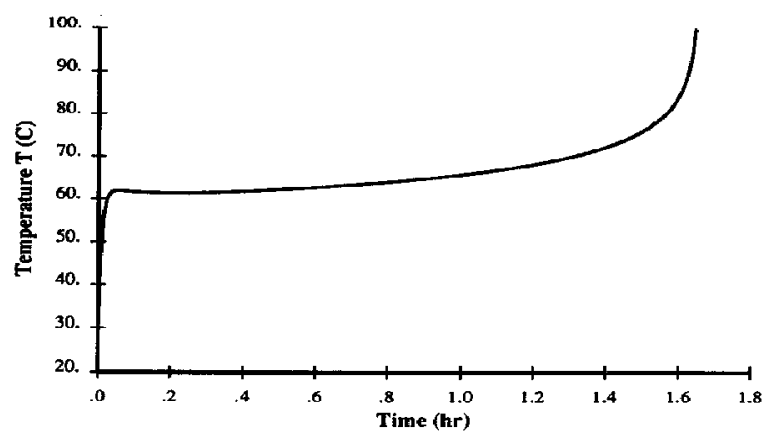

Fig. 9. Temperature profile using control law (40) with $10 \%$ error in $\left[M_{1}\right](0)$.

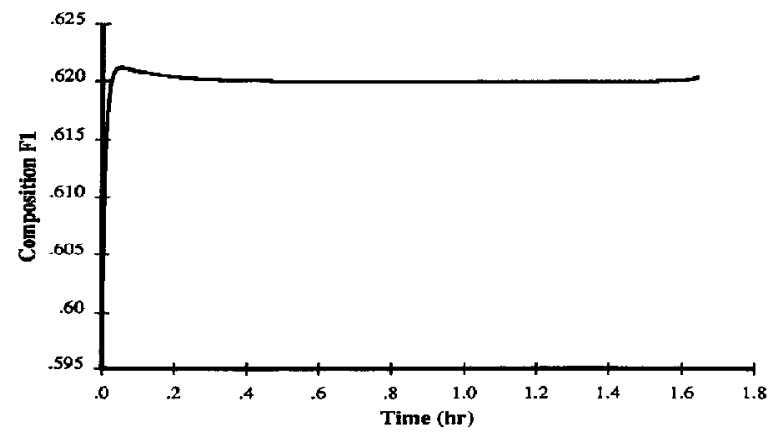

Fig. 10. $F_{1}$ and $\hat{F}_{1}$ profiles using control law (40) with $10 \%$ error in $\left[M_{1}\right](0)$.

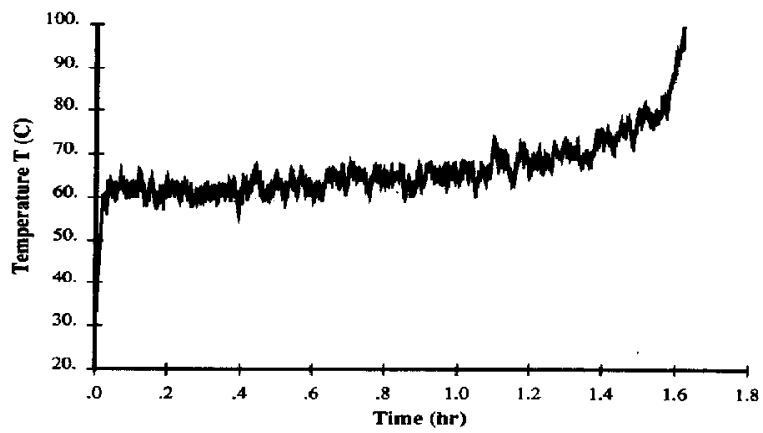

Fig. 11. Temperature profile using control law (40) with noise $(\mathrm{SD}=500)$ in $Q$.

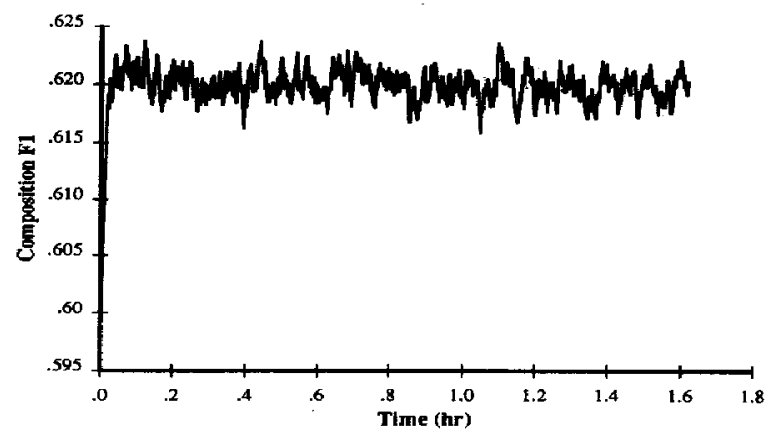

Fig. 12. $F_{1}$ and $F_{1}$ profiles using control law (40) with noise $(\mathrm{SD}=500)$ in $Q$. the initial error in $\zeta_{1}$ is $1 \%$. In industrial situations the initial monomer concentrations can be measured quite accurately, and the initial monomer 1 fraction can therefore be equally accurate. It is important to note that the error increases with conversion, not with time. Thus only for extremely high conversions $(>90 \%)$ will there be any significant drift in the composition. In the third simulation (Figs 9 and 10) a $10 \%$ error was introduced in the initial condition of $\left[M_{1}\right]$. Since $F_{1}$ is not explicitly dependent on $\left[M_{1}\right]$ an offset in the response was not encountered. In the fourth case (Figs 11 and 12) white noise was added to $Q$ with a SD of 500 . The noise is clearly attentuated by the control system. Note that in all four cases the overshoot from using a startup temperature is very small and dies out quickly.

Figures 13-20 show the resulting temperature and $F_{1}$ profiles for four different cases using the control law in terms of $T$ [equation (41)]. Using this control strategy it is not necessary to estimate $F_{1}$. For all four simulations $K_{\mathrm{c}}=0.5$ and $\tau_{I}=50$. In the first run (Figs 13 and 14) there were no disturbances. In the second case (Figs 15 and 16), a $1 \%$ error was introduced in the initial condition of $\zeta_{1}, F_{1}(t)$ was not equal to 0.62 when $\zeta_{1}(0)$ was in error in the same manner and for the same reason as when using the previous control law (40). In the third case (Figs 17 and 18), a 10\% error was introduced in the initial condition of $\left[\mathrm{M}_{1}\right]$. In the fourth simulation (Figs 19 and 20 ) white noise was added to $Q$ with a SD of 500 . The noise is again clearly attentuated by the control system. Note that in all four cases the overshoot from using a startup temperature is very small and dies out quickly. Although the response is somewhat better for the runs where $T$ was used directly in the control law, it should not be inferred that this method is better. Since it was beyond the scope of this work to determine the best controller settings for each method, all that can be determined is that both strategies will give a good response.

\section{CONCLUSIONS}

Nonlinear control has been proposed as a potential approach for trajectory tracking in batch processes. A general nonlinear feedforward-feedback control methodology was derived for systems of relative order one. The temperature control of a copolymerization reactor to obtain constant composition was chosen as an example to illustrate the use of this method. For this system, the feedforward part consists of two terms. The first term cancels the instantaneous heat of reaction and the other provides the necessary temperature gradient to keep the composition at the desired level. Simulations show the ability of this method to track the required nonlinear profiles for this system. 


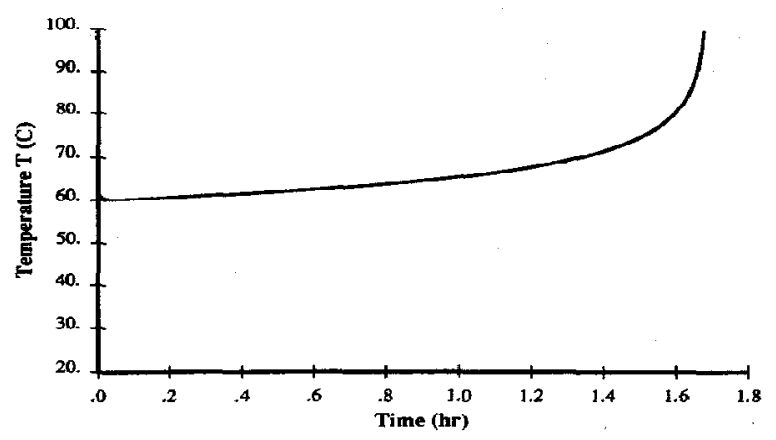

Fig. 13. Temperature profile using control law in terms of $T$ [equation (41)] with no disturbance.

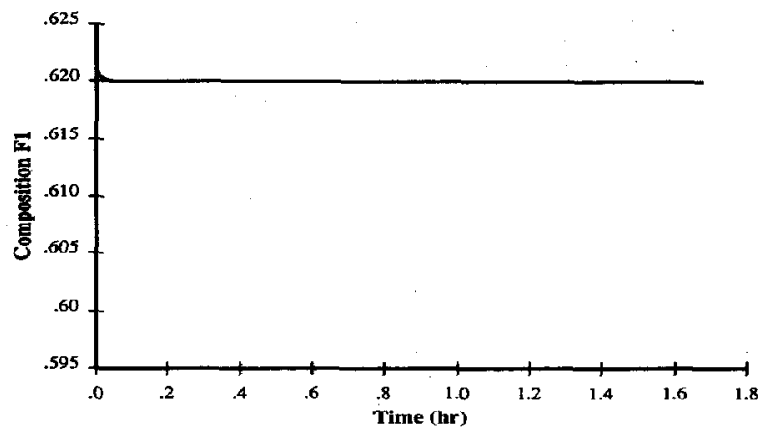

Fig. 14. F, profile using control law in terms of $T$ [equation (41)] with no disturbance.

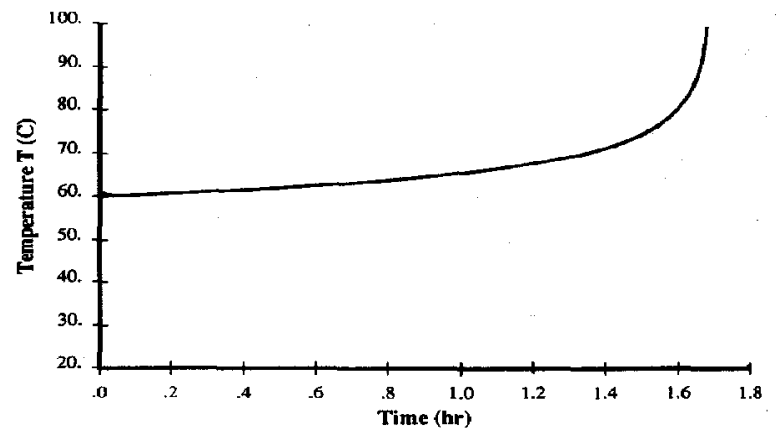

Fig. 15. Temperature profile using control law (41) with $1 \%$ error in $\zeta_{1}(0)$.

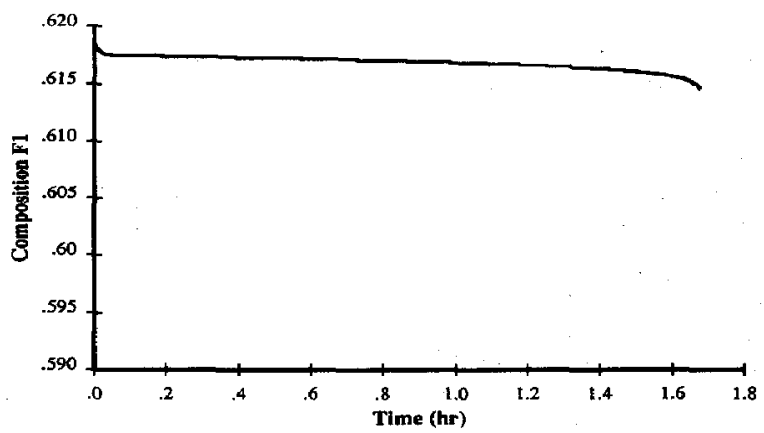

Fig. 16. $F_{1}$ profile using control law (41) with $1 \%$ error in $\zeta_{1}(0)$.

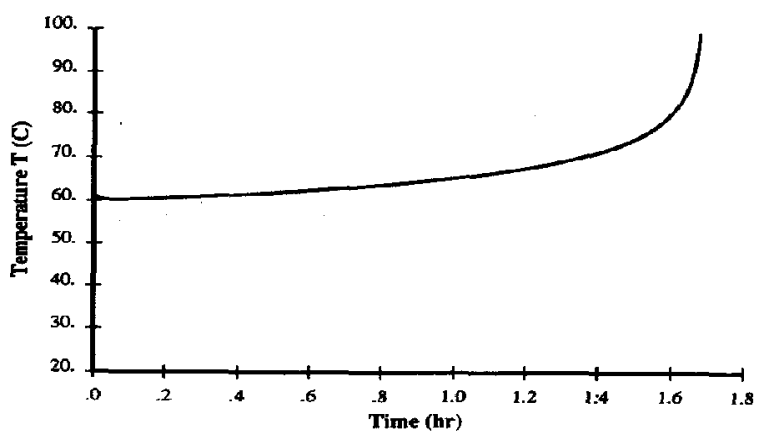

Fig. 17. Temperature profile using control law (41) with $10 \%$ error in $\left[\mathrm{M}_{1}\right](0)$.

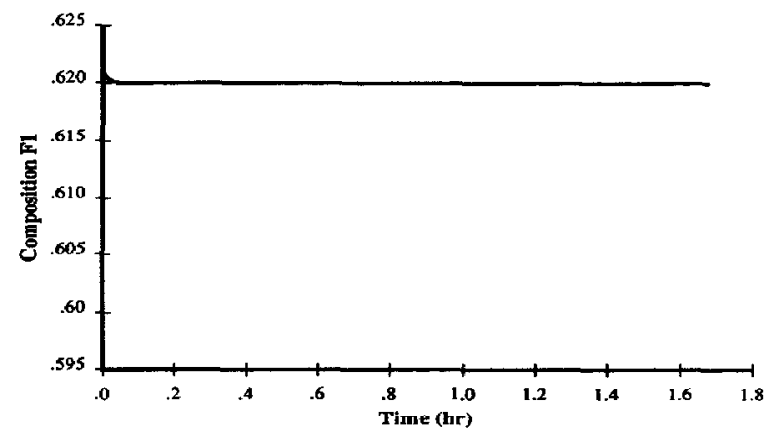

Fig. 18. $F_{1}$ profile using control law (41) with $10 \%$ error in $\left[\mathrm{M}_{1}\right](0)$.

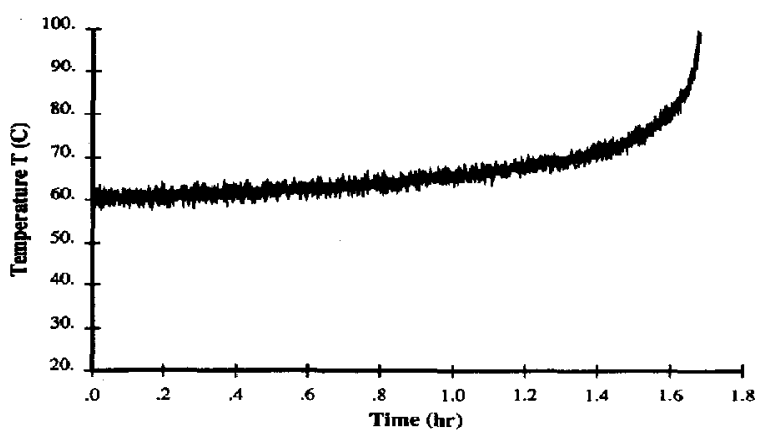

Fig. 19. Temperature profile using control law (41) with noise $(S D=500)$ in $Q$.

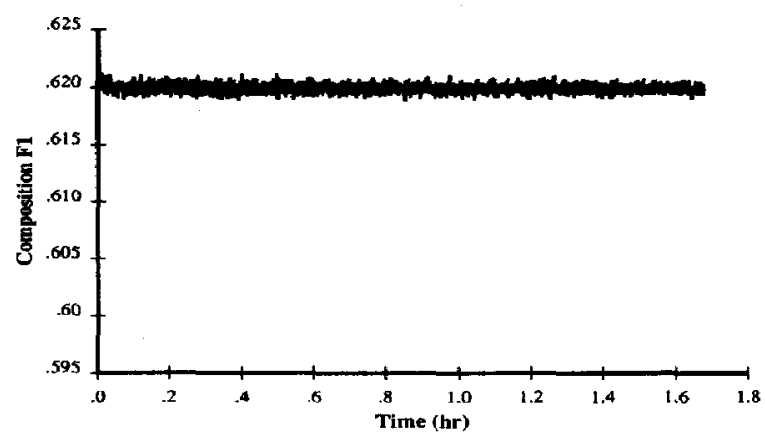

Fig. 20. $F_{1}$ profile using control law (41) with noise $(\mathrm{SD}=500)$ in $Q$. 


\section{NOMENCLATURE}

$A=$ Preexponential factor, $1 \mathrm{~mol} \mathrm{~s}{ }^{-1}$

$C_{\mathrm{prn}}=$ Mean heat capacity per unit volume, cal $1^{-1} \mathrm{~K}^{-1}$

$C_{\mathrm{M}_{1}}=$ concentration of monomer 1 in inlet stream for semibatch case, mol $^{-1}$

$d=$ Disturbance

$E=$ Activation energy, $\mathrm{kcal} \mathrm{mol}^{-1}$

$F_{1}=$ Mol fraction of monomer 1 units in copolymer

$F_{i}=$ Inlet flowrate in semibatch case, $1 \mathrm{~s}^{-1}$

$[I]=$ free radical initiator concentration, $\mathrm{moll}^{-1}$

$k_{\mathrm{DI}}=$ Rate constant for decomposition of free radical initiator

$k_{p i j}=$ Propagation rate constant for addition of monomer of type $j$ to radical of type $i$

$k_{i j}=$ Termination rate constant for bimolecular reaction of radicals of type $i$ and $j$

$K_{\mathrm{c}}=$ Controller proportional gain

$\left[M_{1}\right]=$ Concentration of monomer $1, \mathrm{~mol} \mathrm{l}^{-1}$

$\left[\mathrm{M}_{2}\right]=$ Concentration of monomer $2, \mathrm{~mol} \mathrm{l}^{-1}$

$Q=$ Heat added to the system

$r=$ Relative order of system

$r_{1}=$ Reactivity ratio $\left(k_{p 11} / k_{p 12}\right)$

$r_{2}=$ Reacivity ratio $\left(k_{p 22} / k_{p 21}\right)$

$\mathbf{R}=$ Ideal gas constant

$\boldsymbol{R}_{i}=$ Rate of decomposition of free radical initiator

$\boldsymbol{R}_{\mathrm{p}}=$ Overall rate of polymerization $\left\{\mathrm{d}\left(\mathrm{M}_{1}+\mathbf{M}_{2}\right) / \mathrm{d} t\right\}$

$u=$ Manipulated variable

$V=$ Volume of batch reactor vessel, 1

$\boldsymbol{x}=$ State

$y=$ Output

Greek symbols

$\Delta E_{1}=$ Activation energy for reactivity ratio $r_{1}$, kcal mol-1

$\Delta E_{2}=$ Activation energy for reactivity ratio $r_{2}$, kcal mol-1 $^{-1}$

$\Delta H_{\text {cop }}=$ Heat of copolymerization, $\mathrm{kcal} \mathrm{mol}^{-1}$

$\phi, \xi_{1}, \xi_{2}, \psi_{1}, \psi_{1}=$ Intermediate variables in copolymerization equations

$\zeta_{1}=$ Mol fraction monomer 1 in polymerizing mass, $\left[\mathbf{M}_{1}\right] /\left[\mathbf{M}_{1}\right]+\left[\mathbf{M}_{2}\right]$

$\zeta_{2}=$ Mol fraction monomer 2 in polymerizing mass, $\left[\mathbf{M}_{2}\right] /\left[\mathbf{M}_{1}\right]+\left[\mathbf{M}_{2}\right]$

$\tau_{1}=$ Reset

Subscripts

$0=$ Initial condition ref $=$ Reference value

sp $=$ Set point value

Superscripts

$$
\wedge=\text { Estimated quantity }
$$

\section{REFERENCES}

Blout E. R., Monomers. Interscience, New York (1949).

Clarke D. W. and P. J. Gawthrop, Implementation and application of microprocessor-based self-tuners. Automatica 17, 233-244 (1981).

Constandinides A., J. L. Spencer and E. L. Gaden Jr, Optimization of batch fermentation processes. Biotech. Bioengng 12, 803, 1081 (1970).

Georgakis C., On the use of extensive variables in process dynamics and control. Chem. Engng Sci. 41, 1471-1484 (1986).

Juba $M$. R. and J. W. Hamer, Progress and challenges in batch process control. Chemical Process Control-CPC III (M. Morari and T. J. McAvoy, Eds) pp. 139-183. Elsevier, Amsterdam (1986).

Jutan A. and A. Uppal, Combined feedforward-feedback servo control scheme for an exothermic batch reactor. Ind. Engng Chem. Process Des. Dev. 23, 597-602 (1984).

Kiparissides C. and S. L. Shah, Self-tuning and stable adaptive control of a batch polymerization reactor Automatica 19, 225-235 (1983).

Kravaris $C$. and $C$. B. Chung, Nonlinear state feedback synthesis by global input/output linearization. AIChE $J l$. 33, 592-603 (1987).

Marroquin G. and W. L. Luyben, Experimental evaluation of nonlinear cascade controllers for batch reactors. Ind. Engng Chem. Fundam. 11, 552-556 (1972).

Marroquin G. and W. L. Luyben, Practical control studies of batch reactors using realistic matehmatical models. Chem. Engng Sci. 28, 993-1003 (1973).

Miyama H. Journal of Polymer Science 54, 32 (1961).

Ray W, H. and C. E. Gall, The control of copolymer composition distributions in batch and tubular reactors. Macromolecules 2, 425 (1969).

Tirrel M. and K. Gromley, Composition control of batch copolymerization reactors. Chem. Engng Sci. 36, 367-375 (1981).

Touloukian Y. S. and C. Y. Ho, Thermophysical Properties of Matter, Vol 6 suppl. IFI/Plenum, New York (1976).

Tsoukas A., M. Tirrell and G. Stephanopoulos, Multiobjective dynamic optimization of semibatch copolymerization reactors. Chem. Engng Sci. 37, 1785-1795 (1982).

Wu R. S. H., Dynamic thermal analyzer for monitoring batch processes. Chem. Engng Prog. 81, 57-61 (1985). 